\title{
An Efficient Branch-and-Bound Solver for Hitting Set
}

\author{
Thomas Bläsius * $^{*} \quad$ Tobias Friedrich ${ }^{\dagger} \quad$ David Stangl $^{\dagger} \quad$ Christopher Weyand *
}

\begin{abstract}
The hitting set problem asks for a collection of sets over a universe $U$ to find a minimum subset of $U$ that intersects each of the given sets. It is NP-hard and equivalent to the problem set cover. We give a branch-and-bound algorithm to solve hitting set. Though it requires exponential time in the worst case, it can solve many practical instances from different domains in reasonable time. Our algorithm outperforms a modern ILP solver, the state-of-the-art for hitting set, by at least an order of magnitude on most instances.
\end{abstract}

\section{Introduction}

Hitting set naturally emerges from many problems appearing in various domains, e.g., transportation 34, model-based diagnosis [28, data profiling [3, or biology [23. Unfortunately, hitting set is NP-hard. In fact, it is among the first 21 NP-complete problems [24].

Beyond its NP-completeness, there is a wide range of theoretic results on hitting set, including exact algorithms [30, approximation results [32, 12, 16, parameterized algorithms [1, 17, and parameterized approximation algorithms [7. Moreover, several variants of the problem have been studied, e.g., weighted variants [17, geometric variants, where the instance represents geometric objects 12 , implicit hitting set, where the instance is not explicitly given but implicitly by an oracle that reveals sets not yet hit [11, 25, and the enumeration variant, where one has to find all inclusion-wise minimal hitting sets instead of just the minimum [18].

Due to its importance for various applications, hitting set has also been studied from a practical perspective. A lot of engineering work has been dedicated to the above mentioned enumeration variant; see the survey by Gainer-Dewar and Vera-Licona [18, for an overview and the paper of Murakami and Uno 26] for the state-of-theart algorithm. For the optimization problem of finding a minimum hitting set, there are results on heuristic algorithms, e.g., [35, 14, as well as heavily parallelized brute-force approaches using GPUs [9, 10].

Concerning clever algorithmic techniques for solving hitting set exactly, there is the seminal work of Weihe 34] proposing two rules for data reduction that perform very well on instances coming from rail net-

\footnotetext{
*Karlsruhe Institute of Technology.

${ }^{\dagger}$ Hasso Plattner Institute.
}

works 4. More recently, Bevern and Smirnov 33. proposed alternative reduction rules for $d$-hitting set (restricting the size of each set to at most $d$ ) and evaluated them on instances coming from the cluster vertex deletion problem. Though reduction rules are a crucial component in designing efficient algorithms, one generally still needs an algorithm to solve the remaining instance. Concerning such an algorithm, the current state-of-theart is somewhat unsatisfactory. In 2000, Caprara, Toth, and Fischetti [8] did an exhaustive study of all prevalent solvers at the time and conclude:

This shows that the state-of-the-art generalpurpose ILP solvers are competitive with the best exact algorithms for $\mathrm{SCP}^{1}$ presented in the literature, and that their performance can sensibly be improved by an external preprocessing procedure. ([] $)$

Later, de Kleer [15] conducted an empirical study on the effect of Weihe's reduction rules 34 in a simple branchand-bound algorithm. However, the algorithm does not outperform a general-purpose ILP solver. To the best of our knowledge, using an ILP solver, potentially after preprocessing, remains the state-of-the-art to this day.

In this paper, we engineer and evaluate a branchand-bound algorithm that beats this state-of-the-art. On our test set of 929 instances where the ILP solver reported a non-zerd ${ }^{2}$ running time, we reach a median speedup factor of more than 25. For three quarters of these instances, we have a speedup of more than one order of magnitude.

The basic building blocks of our branch-and-bound algorithm are bounds on the solution size and data reduction rules. They are described in Section 2. We note that most bounds and reduction rules we use have been considered before, either for hitting set or in a different context. For the different lower bounds we give a theoretical analysis that completely characterizes how they relate to each other; see Section 2.3. In Section 3 we specify our overall algorithm and provide details on how to efficiently implement it. Our evaluation in Section 4 is based on 4256 instances from different

\footnotetext{
${ }^{1} \mathrm{SCP}$ stands for "set cover problem", which is equivalent to the hitting set problem.

${ }^{2}$ Gurobi reports running times below 0.01 as 0 .
} 
domains. Beyond the overall running time of our algorithm, we give a detailed evaluation of how much the different building blocks contribute to the final result. Our implementation is publicly availabl $\$^{3}$

\section{Basic Building Blocks}

In this section we describe lower and upper bounds as well as reduction rules and introduce the hitting set problem and our notation.

2.1 Problem Definition. Formally, a hypergraph $\mathcal{F}$ is a set family over a vertex set $V$, i.e., every $F \in \mathcal{F}$ is a subset of $V$. We call these elements $F \in \mathcal{F}$ hyperedges. For brevity, we will refer to them as edges. The number of vertices in the hypergraph is $|V|$, and likewise the number of edges $|\mathcal{F}|$. Additionally, we use $\|\mathcal{F}\|$, called the hypergraph size, to refer to the sum of all edge sizes, i.e., $\|\mathcal{F}\|=\sum_{F \in \mathcal{F}}|F|$. For a vertex $v \in V$, we denote the set of edges containing $v$ as $\mathcal{F}(v)$. We call $\operatorname{deg}(v)=|\mathcal{F}(v)|$ the degree of $v$. Note that the sum of vertex degrees is equal to $\|\mathcal{F}\|$.

We say that a vertex hits an edge if it is contained in it. Based on this, we call a vertex subset $H \subseteq V$ a hitting set of $\mathcal{F}$ if all edges in $\mathcal{F}$ are hit by at least one vertex in $H$. Formally, $H \subseteq V$ is a hitting set of $\mathcal{F}$ if and only if $\forall F \in \mathcal{F}: H \cap F \neq \emptyset$. We call a hitting set minimum if no smaller hitting set for the same hypergraph exists. We refer to a hitting set as minimal if it contains no other hitting set as proper subset. The hitting set problem asks for a minimum hitting set of a given hypergraph.

2.2 Upper Bounds. For the upper bound, we use the simple greedy algorithm of repeatedly picking the vertex with the highest degree. This results in a $\log n$ approximation, works well in practice, and runs in linear time [20, 31. We note that there are multiple LP-based upper (and also lower) bounds for which we refer to the overview by Caprara 8 .

2.3 Lower Bounds. In contrast to upper bounds, good lower bounds are harder to achieve, but crucial for the pruning. Here we describe five lower bounds, some of which have been used for hitting set or other problems. Moreover, we prove a complete characterization of how the lower bounds relate to each other.

max-degree bound The max-degree bound uses that each vertex hits at most $d_{\max }$ many edges, where $d_{\max }$ is the highest vertex degree. Thus, at least $\left[\frac{|\mathcal{F}|}{d_{\max }}\right]$ vertices are required to hit all edges.

\footnotetext{
https://github.com/Felerius/findminhs
}

sum-degree bound Let $d_{1}, \ldots, d_{n}$ be the vertex degrees in descending order. Since vertices can only be chosen once, the max-degree bound can be improved to the smallest $k$ for which $\sum_{i=1}^{k} d_{i} \geq|\mathcal{F}|$.

efficiency bound Consider any solution $S$. Let each vertex $v \in S$ charge its cost onto the edges it hits. That is, each edge $F \in \mathcal{F}(v)$ is charged $1 / \operatorname{deg}(v)$ by $v$. The size of the solution can now be expressed as the sum of the cost of all edges, i.e., $|S|=$ $\sum_{F \in \mathcal{F}} \sum_{v \in S \cap F} 1 / \operatorname{deg}(v)$. The efficiency bound assumes the lowest cost for each edge individually, yielding $\left\lceil\sum_{F \in \mathcal{F}} \min _{v \in F} \frac{1}{\operatorname{deg}(v)}\right\rceil$ as a lower bound.

packing bound A set $P$ of pairwise disjoint edges constitutes a lower bound, because each vertex appears in at most one of those edges. Thus at least $|P|$ vertices are required to cover them. Finding the best packing bound is actually an independentset problem on the intersection graph of the edges $\mathcal{F}$. Using an independent set of conflicts as a lower bound is a known technique applied by recent solvers for other hard problems [19].

sum-over-packing Any packing $P$ can be used to strengthen the sum-degree bound, as the packing requires to select $|P|$ vertices to cover the edges in $P$, which might not be the vertices of highest degree that the sum bound would use otherwise. In the remainder, we focus on how many edges of $\mathcal{F} \backslash P$ we cover. The vertices selected to cover $P$ can cover at most $b_{P}=\sum_{F \in P} \max _{v \in F}(\operatorname{deg}(v)-1)$ edges in $\mathcal{F} \backslash P$. If $b_{P}$ is smaller than $|\mathcal{F} \backslash P|$, we have to pick additional vertices to cover the remaining $|\mathcal{F} \backslash P|-b_{p}$ edges. To this end, let $d_{1}, \ldots, d_{n}$ be the descending vertex degrees in $\mathcal{F} \backslash P$, excluding the vertex of highest degree of each edge in $P$ (these vertices have already been selected and cannot be selected twice). With this, the improved sum bound is $|P|+k$ where $k \geq 0$ is the smallest number for which $\sum_{i=1}^{k} d_{i} \geq|\mathcal{F} \backslash P|-b_{P}$.

A lower bound $a$ dominates another bound $b$, if $a \geq b$ for all problem instances. If a bound has multiple choices (e.g., the packing bounds), we consider the choice that leads to the highest bound. Two bounds are incomparable if neither dominates the other, i.e., there is an instance where $a<b$ and another instance where $a>b$. Figure 1 shows the relations between the lower bounds stated in the following lemmas. Their proofs can be found in section A

LEMMA 2.1. The sum-over-packing dominates the sumdegree and the packing bound. The sum-degree bound 


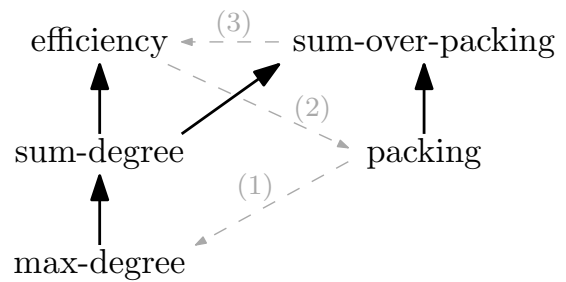

Figure 1: Relations between lower bounds. A bold arrow from $a$ to $b$ means that $a$ is dominated by $b$ (Lemma 2.1). Dashed arrows are labeled as in Lemma 2.2 and indicate that there exists an instance where $a$ is smaller than $b$. Note that for any pair of bounds, there is either a directed bold path (indicating dominance) or a directed path containing exactly one dashed edge (indicating non-dominance).

dominates the max-degree bound and is dominated by the efficiency bound.

Lemma 2.2. The packing bound is incomparable with the max-degree, sum-degree, and efficiency bound. The efficiency bound is incomparable with the sum-overpacking bound.

2.4 Reduction Rules. Our algorithm uses the following reduction rules. The domination rules were first described by Weihe [34. The costly discard rule is a standard technique in branch and bound but has, to the best of our knowledge, not yet been used for hitting set. The unit edge rule is widely known and stated, e.g., by Shi and Cai [30].

Unit Edge Rule. If there is an edge of size one, then pick the contained vertex.

Edge Domination Rule. If there are two edges $e_{1}, e_{2}$ with $e_{1} \subseteq e_{2}$, then delete $e_{2}$.

Vertex Domination Rule. If there are two vertices $v_{1}, v_{2}$ such that $\mathcal{F}\left(v_{1}\right) \subseteq \mathcal{F}\left(v_{2}\right)$, then delete $v_{1}$.

Costly Discard Rule. If discarding a vertex raises the lower bound to or above the current upper bound, then pick this vertex.

We do not consider the complement of the costly discard rule (costly inclusion) because including a vertex cannot raise the packing lower bound by more than one. The rule could only take effect when the upper and lower bound differ by one, in which case the instance is almost solved anyway.

We note that Shi and Cai 30. proved that branchand-bound runs in time $O\left(1.23801^{n}\right)$ when using the first three of the above reduction rules in addition to two rules based on edges of size two. We omitted these two rules from our solver, as they rarely take effect.

\section{The Branch-and-Bound Algorithm}

In this section we describe the structure and efficient implementation of our solver as outlined in Algorithm 1 . In every step, our algorithm branches on the inclusion or exclusion of a vertex in the solution, thereby creating two new instances that are solved recursively. Before each branch, we apply the follow two steps. First, an approximate solution for the current instance is found using a greedy algorithm. The best found solution so far represents the current upper bound and is globally maintained as a result of the algorithm. It is used to prune branches where no better solution can be achieved. Second, reduction rules are repeatedly applied until either no reduction is possible or a lower bound allows the current branch to be pruned.

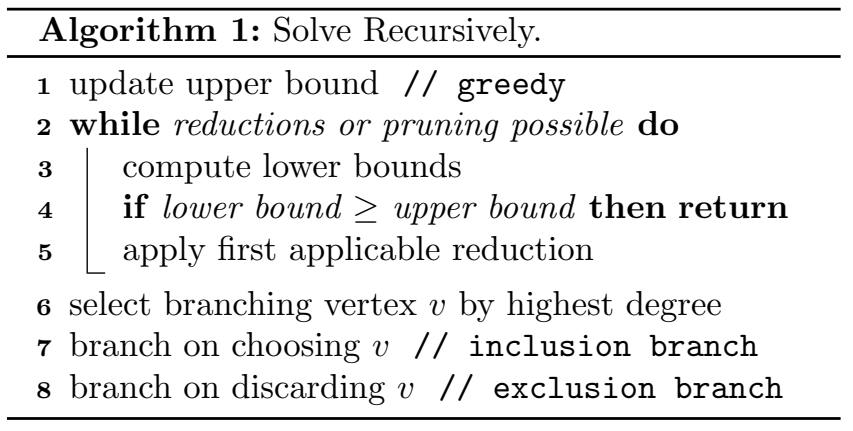

3.1 Operation Summary and Reduction Order. Initially we compute a greedy upper bound in time $O(\|\mathcal{F}\|)$ but do not repeat it in the reduction loop. Then, reductions and bounds are checked one after another (Algorithm 1, line 2-5). They are processed in ascending order by runtime to prevent expensive operations when possible. After one reduction is applied, the search starts from the top of the list again. In general, lower bound pruning happens before reductions. Although the max-degree bound is dominated by the efficiency bound, we include it due to the lower computational complexity. The order of lower bounds and reductions is as follows.

1. max-degree bound in $O(|V|)$

2. efficiency bound in $O(\|\mathcal{F}\|)$

3. packing bound in $O(|| \mathcal{F}||+|\mathcal{F}| \log |\mathcal{F}|)$

4. sum-over-packing bound in $O(\|\mathcal{F}\|)$

5. unit edge rule in $O(|\mathcal{F}|)$ 
6. costly discard rule with efficiency bound updates for all vertices in $O(\|\mathcal{F}\|)$

7. costly discard rule with packing updates for all vertices in $O(\| \mathcal{F}||+|\mathcal{F}| \log |\mathcal{F}|)$

8. costly discard rule with repack for 3 vertices in $O(|| \mathcal{F}||+|\mathcal{F}| \log |\mathcal{F}|)$

9. edge domination rule in $O(|\mathcal{F}| \cdot|| \mathcal{F}||)$

10. vertex domination rule in $O(|V| \cdot|| \mathcal{F}||)$

In the following, we discuss the branching strategy and implementation details of the instance representation, the bound computation, and the reduction rules.

3.2 Branching Strategy. As mentioned above, we branch on the inclusion or exclusion of a verticex. The remaining degrees of freedom are the vertex to branch on and the order in which the two branches are processed. We found the latter to be irrelevant while the former crucially affects search space and performance. Our solver always branches on the vertex with highest degree in the remaining instance and processes the inclusion branch first.

3.3 Instance Representation. During the algorithm the instance needs to be updated regularly. To avoid copying the instance, we maintain one data structure representing the current instance throughout the algorithm. There are three places in the algorithm where the instance is modified. First, the greedy algorithm iteratively deletes vertices. Second, the reduction rules reduce the instance. Third, when branching, a vertex is excluded (it is deleted) or is included (it and all its edges are deleted). In all cases, changes have to be rolled back appropriately.

To support these operations, we maintain the vertices of each edge and the edges of each vertex in sorted order at all times. Maintaining the order speeds up setlike operations, e.g., union of two edges, and is required by the reduction rules for edge and vertex domination.

For this, we implement a data structure called ordered subset list that manages a subset $S \subseteq\left\{s_{1}, \ldots, s_{n}\right\}$ of $n$ strictly-ordered objects $s_{1}<s_{2}<\cdots<s_{n}$. Assuming the $s_{i}$ are sorted in advance, it supports the following operations.

\begin{tabular}{rlc}
\hline Name & Description & Time \\
\hline $\operatorname{init}()$ & Initialize $S=\left\{s_{1}, \ldots, s_{n}\right\}$ & $O(n)$ \\
$\operatorname{del}(i)$ & Delete $s_{i}$ from $S$ & $O(1)$ \\
undo() & Undo last (not undone) deletion & $O(1)$ \\
iter() & Traverse $S$ in increasing order & $O(|S|)$ \\
iterrev() & Traverse $S$ in decreasing order & $O(|S|)$ \\
\hline
\end{tabular}

This can be implemented by storing the subset $S$ itself in a doubly-linked list. Additionally, we have an array $A$ that points for each $i$ to the list entry corresponding to $s_{i}$. When deleting an element from $S$, its list entry can be found in constant time via $A$. It is removed from the list, but the list item itself remains in memory and $A$ keeps the pointer to it. To allow for later reinsertion, we maintain a stack of indices of deleted list entries. As the list entry itself has not been modified at the time of deletion, its previous and next entries are intact and thus we can reinsert it into the list in constant time in the position it was before its deletion.

3.4 Upper Bound Computation. The greedy algorithm picks the highest degree vertex and deletes it and its edges from the instance until all edges are hit. Since modifications, i.e., deleting edges and vertices, are done in time linear in the number of changes (see Section 3.3. this totals to at most linear time until a hitting set is found. Finding the vertex with highest degree in each step is done with a bucket heap [13, 31] that stores the vertex degrees. The data structure allows constant time operations due to the limited range of the stored values. Since degrees are only lowered during the procedure and the total vertex degree is $\|\mathcal{F}\|$ the greedy algorithm takes linear time in the size of the instance.

3.5 Packing Bound Computation. Finding a maximum packing of disjoined edges is an independent set problem and thus computationally expensive. Recent solvers for the quasi-threshold editing and cluster editing problem, which use the same idea of packing conflicts, apply the min-degree heuristic to find a good packing [19, 22]. In our context, the degree in the conflict graph of an edge $F$ from the original instance would be the number of other edges that share at least one vertex with $F$. We approximate this and sort all edges by $\sum_{v \in F} \operatorname{deg}(v)$ in ascending order. Then, we go through the edges and add the current edge to the packing if possible. When adding an edge to the packing, each contained vertex is marked. An edge $F$ can be added if all contained vertices are unmarked, which can be checked in $|F|$. In total, the initial packing is computed in $O(\| \mathcal{F}||+|\mathcal{F}| \log |\mathcal{F}|)$.

We implemented the 2-improvement heuristic for independent set to grow the packing [2]. The heuristic is a local search that repeatedly tries to replace an element from the packing with two new ones. Although this technique is effective [19, we found it to be too slow and too rarely applicable to justify the high computational cost (see Figure 5). Our implementation runs in $O(|P|$. $\| \mathcal{F}||)$ per improvement where $P$ is the current packing. 
3.6 Efficient Costly Discard Rule. The costly discard rule states that a vertex must be picked if discarding it raises some lower bound to or above the current upper bound. That is, if we were to branch on that vertex, the exclusion branch would be pruned immediately. The rule has two degrees of freedom: first, the vertex it is applied to and, second, the lower bound that is used. For maximum effectiveness of the reduction, we would like to check the rule for all vertices and lower bounds. However, computing all lower bounds from scratch $|V|$ times is computationally expensive. We restrict it to the efficiency and packing bound. In the following, we discuss how to compute these bounds efficiently for all vertices at once.

Costly Discard with Efficiency Bound. For the efficiency bound, checking the costly discard rule for all vertices at once can be done in $O(\|\mathcal{F}\|)$ as follows. First, the efficiency bound is computed for the current instance. While doing so, for each edge the two vertices with highest degree are saved. When a vertex $v$ would be discarded from the instance, only edges $v$ is contained in can change their contribution to the bound. Such an edge $F$ changes the contribution $\min _{u \in F} 1 / \operatorname{deg}(u)$ only if $v$ was the vertex with highest degree in $F$. In this case the contribution depends on the vertex with second highest degree in $F$, which was identified earlier. In total, discarding $v$ changes the contribution of at most $\operatorname{deg}(v)$ edges that can be updated in constant time each. Over all vertices this sums up to $\|\mathcal{F}\|$.

Costly Discard with Packing Bound. For the packing bound, a similar approach of dynamically updating a packing bound $|V|$ times can be used to check the rule for all vertices and constitutes item 7 in Section 3.1. Discarding a vertex $v$ removes it from all edges. The edges that are relevant are those that intersect the union of the current packing exactly in vertex $v$. That is, they could now be included in the packing after $v$ 's removal. We say that such an edge is blocked by vertex $v$. Each edge is blocked by at most one vertex. After the initial packing is constructed, we create for each vertex $v$ a list of edges that are blocked by this vertex and sort each list individually by the highest degree of a contained vertex (excluding $v$ ). These lists are found and sorted in $O(|| \mathcal{F}||+|\mathcal{F}| \log |\mathcal{F}|)$. When checking if a vertex qualifies for the costly discard rule with the packing bound, we traverse the list of blocked edges for this vertex and greedily add them to the packing if possible. After the rule is checked, we remove the added edges to restore the initial packing. Since each edge $F$ is in at most one list and can be added to the packing in time $O(|F|)$, the costly discard rule can be checked for all vertices in time $O(\|\mathcal{F}\|)$ when using the lists. The creation of the lists dominates the running time with
$O(|| \mathcal{F}||+|\mathcal{F}| \log |\mathcal{F}|)$, which is the same as the time it takes to compute the initial packing.

Unfortunately, the updated packings are worse than if they were computed from scratch. Therefore, we additionally choose the $c$ vertices of highest degree, for which we check the costly discard rule with a completely new packing each (see item 8 in Section 3.1). Our experiments in Section 4.3 suggest that $c=3$ is a reasonable choice.

3.7 Efficient Domination Rules. The domination rules can be checked naively by comparing each set with all others to find inclusions. This implies a running time of $O(|V| \cdot|| \mathcal{F}||)$ and $O(|\mathcal{F}| \cdot|| \mathcal{F} \|)$ which is quadratic in the number of edges or vertices, respectively. In fact, under the strong exponential time hypothesis the reduction cannot be done in less than quadratic time in the worst case 6. In practice, however, the sub- and superset reductions can be sped up significantly using set tries, a data structure described by Savnik [29].

A set trie manages a collection $\mathcal{T}$ of sets over $[n]$ and supports the following operations which require that the given sets are sorted and can be traversed in linear time.

\begin{tabular}{rlc}
\hline Name & Description & Time \\
\hline $\operatorname{add}(S)$ & add $S$ to $\mathcal{T}$ & $O(|S|)$ \\
hasSubset() & does $\mathcal{T}$ contain a & $O(|S|+\|\mathcal{T}\|)$ \\
& subset of $S$ & \\
hasSuperset() & $\begin{array}{l}\text { does contain a } \\
\text { superset of } S\end{array}$ & $O(|S|+\|\mathcal{T}\|)$ \\
\hline
\end{tabular}

For the dominated edge rule we create an empty set trie and iterate through the edges in increasing order of their size. For each edge, we check whether a subset of it exists in the set trie. If so, the edge is dominated. Otherwise the edge is added to the set trie. Note that this process can be continued after the first dominated edge to find all of them.

For the dominated vertex rule, the process is similar. An empty set trie is created that stores sets of edges. Then, vertices are iterated in decreasing order of their degree. Recall that $F(v)$ is the set of edges containing the vertex $v$. If the set trie contains a superset of $F(v)$, the vertex $v$ is dominated. Otherwise, $F(v)$ is added to the set trie.

\section{Evaluation}

In this section, we evaluate our branch-and-bound algorithm experimentally. First, in Section 4.2, we compare its run time to the state-of-the-art ILP-solver Gurobi 21] and analyze how much time is spent in which part of the algorithm. In Section 4.3 , we investigate details regarding the performance and effectiveness 


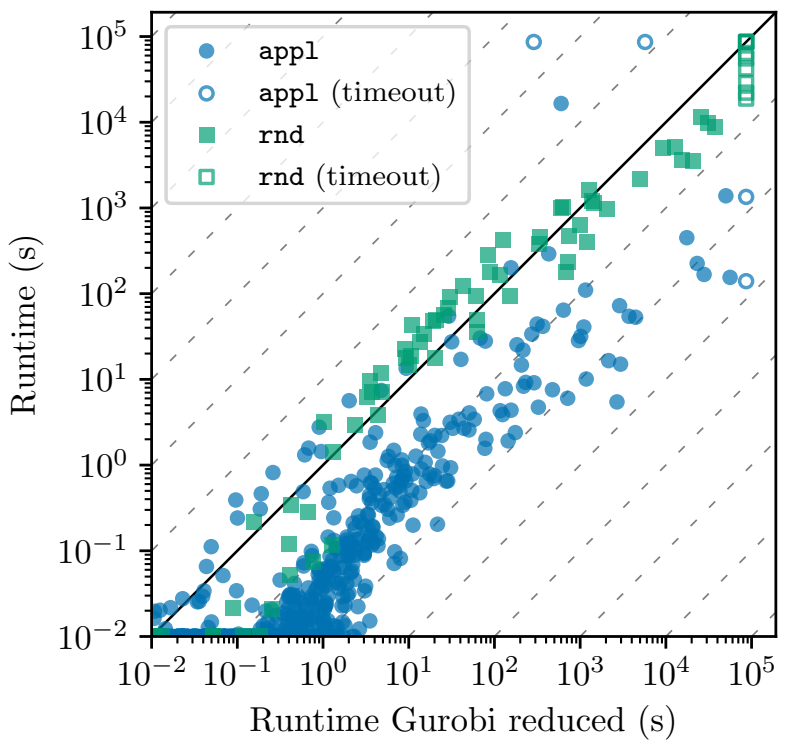

Figure 2: Run time of our solver compared to Gurobi with preprocessing. Times are rounded up to 0.01s.

of lower bounds, upper bounds, and reduction rules to evaluate the used techniques as well as to substantiate our design decisions.

4.1 Experimental Setup. Our implementation is written in the Rust programming language and is available in our public GitHub repository along with all datasets, logs, run results, and evaluation scripts. All auxiliary packages, including the versions used, are listed in the repository as part of the Cargo project format. For the evaluation, we used version 1.53.0 of the Rust compiler with link-time optimization enabled. The experiments were run on a Gigabyte R282-Z93 (rev. 100) server at $2.6 \mathrm{GHz}$ base speed with $1024 \mathrm{~GB}$ DDR4 $(3200 \mathrm{MHz})$ memory. Runs had a timeout of $24 \mathrm{~h}$.

We use instances from four sources.

UCC [3] contains 134 instances, two for each of 67 databases. In the first type of instance, the hitting sets correspond to the unique column combinations of the database. The second type of instances are the transversal hypergraphs of the first type.

CVD [33] contains cluster vertex deletion instances, derived from weighted graphs of protein similarities 27, 5. In the reduction step from weighted graphs to unweighted graphs, we use all edges

\footnotetext{
${ }^{4}$ https://github.com/Felerius/findminhs
}

with non-negative weights. This is consistent with the code linked in the paper of Bevern and Smirnov 33, but differs from the statement in the paper itself, which only uses edges with positive weights. Like the authors, we restict us to hypergraphs with at most $10^{6}$ edges, resulting in a total of 3952 instances.

EN1 [26] contains 159 instances that were previously used to evaluate algorithms for enumerating minimal hitting sets. These contain several classes of instances, including real-world and generated instances. The original data set contains 172 instances of which we omitted 13 whose size $\|\mathcal{F}\|$ exceeds $3 \cdot 10^{7}$, as the RAM required to run experiments on them proved to be prohibitive.

EN2 [18] contains eleven additional instances that have been used to evaluate enumeration algorithms. Five of them are derived from metabolic reaction networks and six from interventions in cell signaling networks.

We distinguish between the randomly generated instances (rnd) from the EN1 dataset and application specific instances (appl) due to their different structure. The instances displayed in the various plots are filtered depending on the context. Figure 2 includes all 4256 instances. Subsequent plots are restricted to the 136 instances (58 rnd, $78 \mathrm{appl}$ ) that finish in 24 hours (excludes 6) and are non-trivial (excludes 4114), that is, instances where our solver runs at least one second in its default configuration. For experiments that compare different configurations, only the instances finishing in all configurations are used. Effected by this is Figure 5 where three instances were dropped due to timeout in some configuration. Additionally, ten instances where dropped in Figure 9 because they had no forced vertices and two instances where dropped in Figure 4 because they never had a branch pruned due to bounds.

4.2 Runtime. Figure 2 shows the run time of our solver in comparison with Gurobi. Gurobi is at version 9.1.2, restricted to a single thread, and without a memory restriction. We note that there are instances where Gurobi uses almost 50GB of memory. Following Caprara 8, instances were reduced with the domination rules before running Gurobi on them. The reduction process is included in the reported run times. Preliminary experiments showed this to be faster than running the ILP alone.

Our solver is significantly faster than Gurobi on non-random instances; on three quarter of non-random instances at least one order of magnitude. Contrasting 


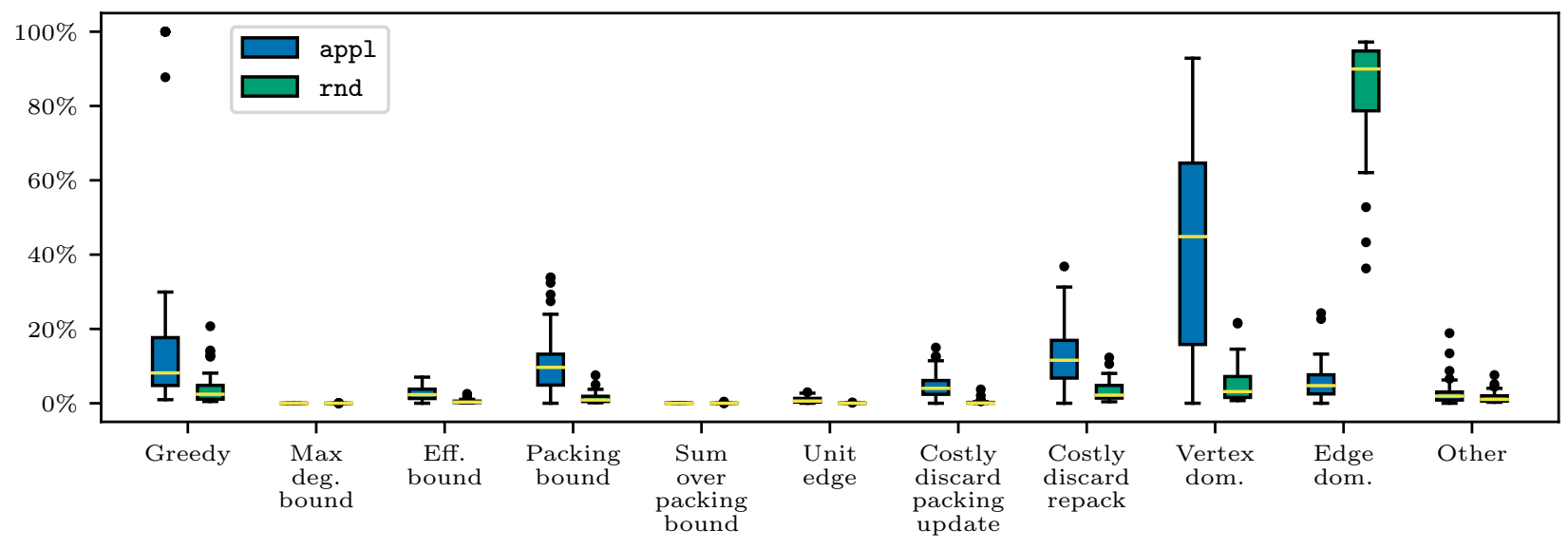

Figure 3: For each instance, the run time share of each operation was measured relative to the total run time of the instance. Note that the time for efficiency costly discard is included in the efficiency bound time.

this, there are only three instances (random and nonrandom) where Gurobi is faster by more than a factor of 4. Run times for random instances are competitive. Gurobi is approximately 1.5 times faster on smaller instances while we are consistently faster on instances that take more than 30 minutes to solve. In total, there are 8 instances that finish in the timeout only for our solver. There are 2 instances that finish just for Gurobi.

Figure 3 shows the fraction of run time that is spent in each step of the algorithm. As expected from the asymptotic considerations in Section 3 , the domination rules dominate the run time although they are executed last and thus avoided when possible. Random instances spent most time in edge domination since they have many edges and few vertices. Non-random instances spend most time in vertex domination. Still, when taking both classes of instances together, the total time spent is spread over different subroutines and, in the median, no individual task takes more than $20 \%$ of the total time. Greedy, packing lower bound, and the costly discard repack reduction rule show comparable times. Although, the repack reduction essentially computes three packings, it is processed later in the loop than the packing lower bound, which explains why the rule does not take three times as much time as the packing. Finally note that the column for other is vanishingly small. It includes, e.g., the instance manipulation and rollbacks as well as logging, timing, and branching.

4.3 Solver Details. In this section we discuss the effectiveness of lower bounds, upper bounds, and reduction rules in detail. Moreover, we provide experimental grounds to argue in favor of our choices regarding the

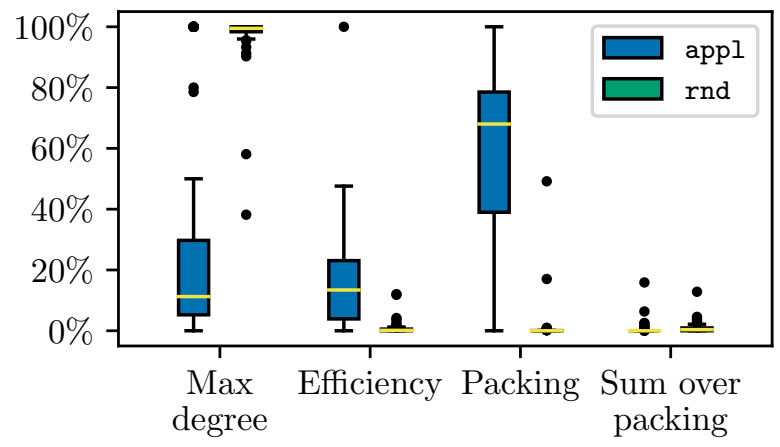

Figure 4: For each instance, the number breaks from the reduction loop split by the responsible bound. Values are relative to the total number of breaks for the instance.

solver configuration. Specifically, we evaluate the set and order of used lower bounds, the number of checked vertices in the costly discard repack rule, the frequency of greedy invocations, and the order in which reductions are applied.

Lower Bound Effectiveness. Figure 4 counts how often each bound was responsible for pruning a branch in the search tree. For random instances, the max-degree bound is sufficient and is responsible for almost all prunes. Application instances make use of several bounds. Max-degree still helps but, excluding a few instances, accounts for less than $30 \%$. The efficiency and the packing bounds then catch branches that are missed by the previous bounds with the packing bound being significantly more successful than the efficiency 


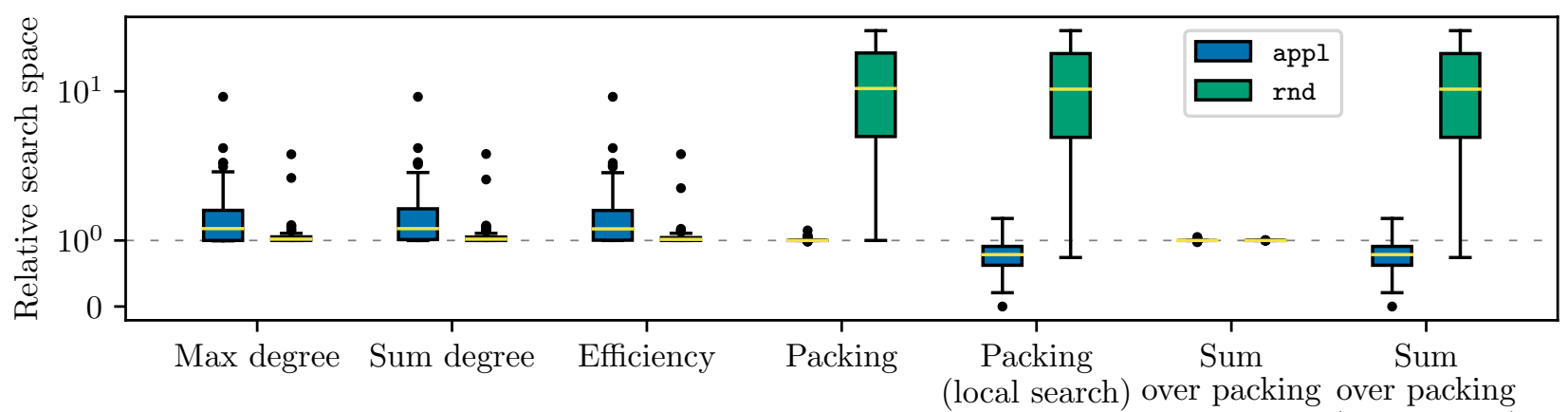

(local search)

Figure 5: Search space when only using a certain bound, relative to default settings. Note the logarithmic y-axis with special handling for zero.

bound despite it being run after it.

For the efficiency bound, the outlier at $100 \%$ is due to an instance with a trivial search space of one node that is pruned by the efficiency bound. Still, there are a few instances where the max-degree bound fails often while the efficiency bound is responsible for $40 \%$ to $60 \%$ of all prunes.

There are even more instances which almost exclusively depend on the packing bound. This can be explained due to the packing bound representing a different approach to obtain the lower bound than the maxdegree or efficiency bound and thus performs well on a different kind of instances. The max-degree bound performs well if the high degree vertices do not share many edges, i.e., if the instance can be covered by selecting few high-degree vertices. On the other hand, many edges containing multiple high-degree vertices makes it possible to have many edges containing only vertices of lower degree, which facilitates large packings.

Regarding the last bound, note that the sum-overpacking bound rarely applies. However, Figure 3 shows that its run time is negligible since the previously computed packing is reused. For some instances the bound actually prunes a significant number of branches.

With the question answered to what extent our chosen lower bounds contribute to pruning, it remains to show that it is their combination and not one bound alone that is responsible for the overall performance. Figure 5 shows the relative search space when using only one bound compared to using our default configuration for the solver. Max-degree, sum-degree, and efficiency all behave similar, that is worse than with all bounds. On the other hand, for random instances only using the packing bound leads to significantly higher search space. These instances, however, are solved easily when using the sum-over-packing instead of the packing alone. In fact, using only sum-over-packing is almost always as good as using the combination of bounds. Nonetheless, it still makes sense to use the other bounds before: The packing bound is free as we have to compute a packing anyway to apply sum-over-packing. Moreover, the simpler bounds can be computed more quickly than a packing (recall Figure 3) but are often sufficient as can be seen in Figure 4.

As mentioned in Section 3.5, we implemented versions of the packing with local search and included them in Figure 5. In the following we consider the influence of adding local search to packing or sum-over-packing. For application instances, adding the local search to the packer or sum-over-packing bound results in a slight improvement over the combination of all other bounds. However, preliminary testing showed local search to be too computationally expensive to justify the reduction in search space it yields. On random instances, adding local search to packing does not change the search space, while adding it to sum-over-packing surprisingly increases the search space. Further investigation revealed that a packing that was augmented with local search is enlarged to the point that taking the highest degree vertex of each edge in the packing constitutes for enough total degree to cover the whole instance. In this case, the sum-over-packing bound is equal to the packing bound.

Upper Bound Effectiveness. The greedy upper bound is used to initialize and, during a run, improve the best solution found so far. Still, the solver could be implemented without greedy at all. The upper bound would then be initialized to contain all vertices in $V$ and updated when the branching reaches a hitting set. Running the greedy subroutine has the benefit that it helps to find solutions before reaching the associated leaf in the search tree and thus facilitates earlier pruning. In the following, we compare four different frequencies 


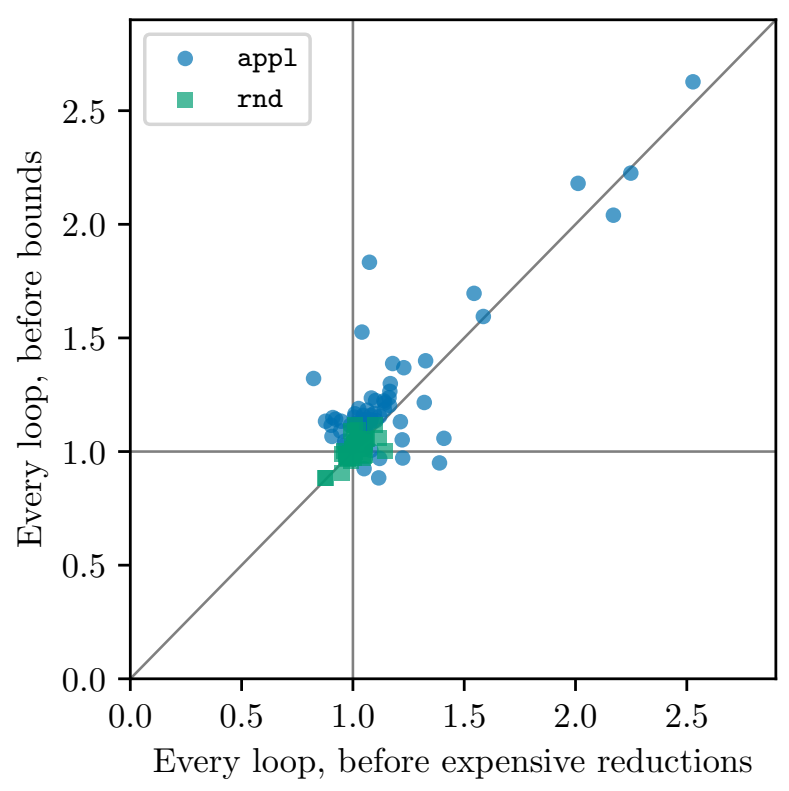

Figure 6: Run times of two non-default greedy modes relative to the run time when using greedy once per node in the search tree. Each instance is a data point.

of running greedy to recompute the upper bound. The alternatives are to not use greedy at all, run greedy once before the reduction loop (which is what we do in the final configuration of our algorithm), run greedy every iteration of the loop (i.e., as item zero in Section 3.1), or to run it every loop just before the expensive reductions (i.e., between items 7 and 8 in Section 3.1).

Figure 6 compares the latter three alternatives. The baseline in the plot (and default configuration for the solver) is to run it once before the loop. The axes show the relative run time compared to this baseline. A point in the lower left quadrant means that the baseline is the worst out of the three; a point in the upper right quadrant that it is best, while the rest ranks it in the middle. Samples are concentrated in the center but slightly tilted to the upper right. Additionally, there are no outliers that favor running greedy every loop while there are outliers that heavily slow down when deviating from our baseline.

Figure 7 shows run times of all alternatives that use greedy compared to not using greedy at all, that is, initializing the upper bound to $V$ and find better solutions only in leaf nodes while branching. Surprisingly, the median favors not using greedy at all but the outliers are heavily in favor of using greedy. Running greedy once before the reduction loop is never slower than 1.5 times the run time of no greedy, 1.02 times slower in the me-

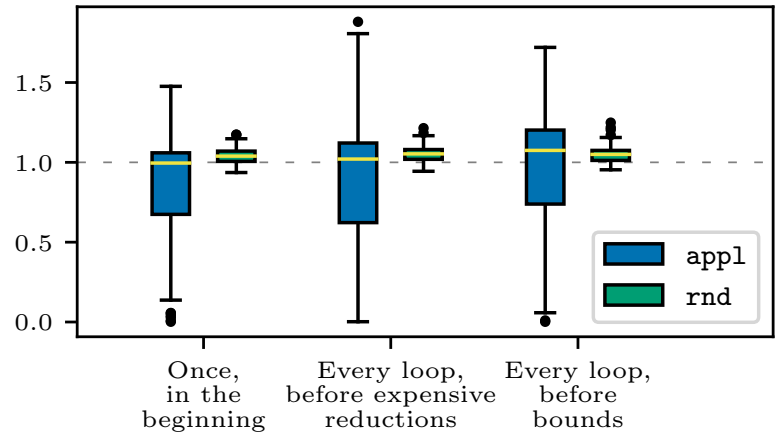

Figure 7: Relative run time for all three greedy modes compared to not using greedy upper bounds.

dian, and up to 600 times faster at best, which makes it a good choice for the final configuration of our algorithm. Note that the benefits of greedy are restricted to application instances. On random instances there are no heavy outliers in favor of any configuration.

Reduction Effectiveness. Before we establish how effective each specific reduction is, we first investigate how often each reduction is actually reached in the reduction loop as shown in Figure 8. Differences between two adjacent reductions express the success rate of the left one. Random instances, again, behave completely different than application specific instances. They almost always execute all reductions, because all reductions before the last one are unsuccessful. For application instances, all rules contribute somewhat. Although the domination rules have the highest run time share (recall Figure 3) they are only executed in 20$30 \%$ of loop iterations for most instances. The most frequent end to an iteration are a successful costly discard rule with packing updates or repacking. These rules are reached in more than $80 \%$ of iterations for most instances and the next rule (vertex domination) is checked $30 \%$ of the time in the median. Note that an iteration only ends in the repack step if the costly discard rule was unsuccessfully checked with a packing update before succeeding through a repack, giving evidence to the usefulness of repacking.

Figure 9 compares the effectiveness of the reductions that force a vertex to be included in the solution. The effectiveness is measured by the number of forced vertices. The unit edge rule and the costly discard rule with the efficiency bound catch almost no vertices compared to the two packing rules. Since they are very easy to compute and find a good amount of application on some instances they are nonetheless worth trying. Above $80 \%$ of forced vertices in application instances 


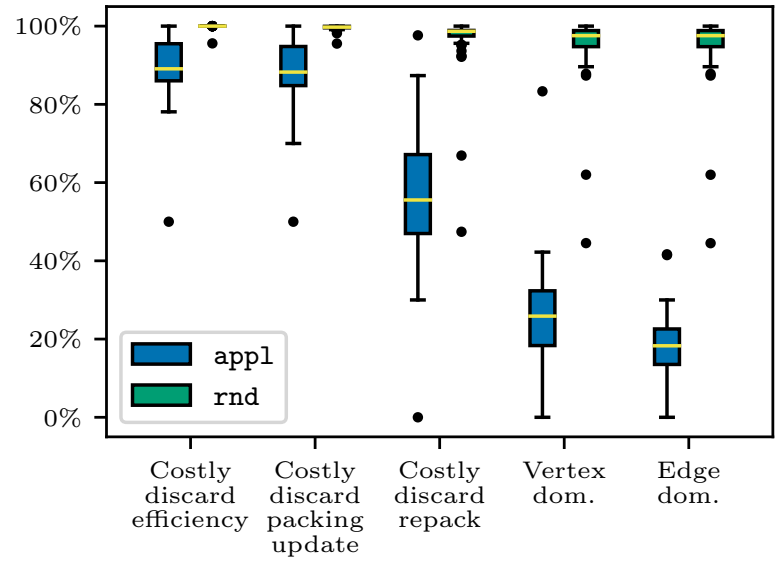

Figure 8: For each instance, the number of reduction loop runs that reach a given reduction. Values are relative to all runs that do not break due to bounds. The forced vertex rule is excluded as it is the first and thus always reached.

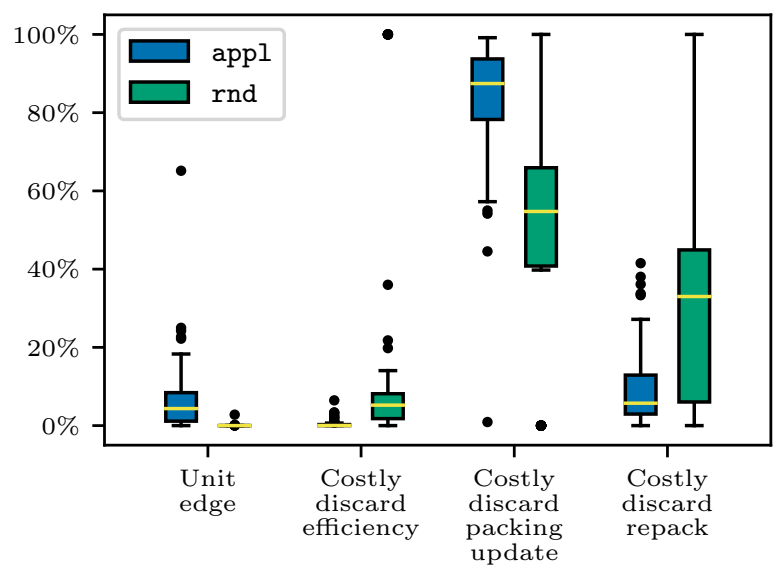

Figure 9: For each instance, the number of found forced vertices by reduction rule. Values are relative to the total number of forced vertices for the instance. are found with the costly discard packing update rule. This constitutes no contradiction to Figure 8 because, although the repack rule is applicable as often, the packing update rule can find multiple forced vertices at once. The fact that the repack rule still finds a large amount of all forced vertices while being applied only after packing update failed to find anything, again, emphasizes that the updated packings are not as good as the packings that are constructed from scratch. The results for random instances are less expressive because only a small number of reductions are applicable, as established in the previous paragraph. Nevertheless, they follow the same trend as the other instances but rate packing update lower and packing repack higher.

One degree of freedom for the repacking is the number of vertices for which we apply it. Recall from Section 3.1 that we repack for the $c=3$ vertices of maximum degree in our final configuration. Figure 10 shows the run time for different values of $c$ relative to the run time when not repacking. One can make two main observations. First, for random instances, the cost of repacking outweighs the gain leading to slightly increasing median run times for increasing $c$. Second, repacking helps significantly on non-random instances but there is no additional gain in repacking more than three times, which has the lowest median. Thus, by using $c=3$, we obtain a good balance between increasing run time for random instances only slightly while obtaining big speedups for some application instances.

\section{Conclusion}

We provide a fast branch-and-bound solver that beats a modern ILP solver, which is the state-of-the-art for solving the minimum hitting set problem. Our implementation provides a baseline for future work in this direction. We explain the basic building blocks of our algorithm — which are lower bounds, upper bounds, and reduction rules - and experimentally evaluate their run time and efficiency to find a good configuration of used rules and bounds. We confirm the effectiveness of Weihes reduction rules noted in previous works. Another crucial part of the algorithm turns out to be the quality of lower bounds. The parameter-dependent Costly Discard Rule builds upon lower and upper bounds and contributes significantly to the performance of our algorithm. Lastly, we find that the algorithm behaves differently on random inputs. In the future, it would be interesting to determine why non-random instances are easier for our solver than random instances and if their structure can be exploited to design even faster algorithms for practical instances.

\section{References}




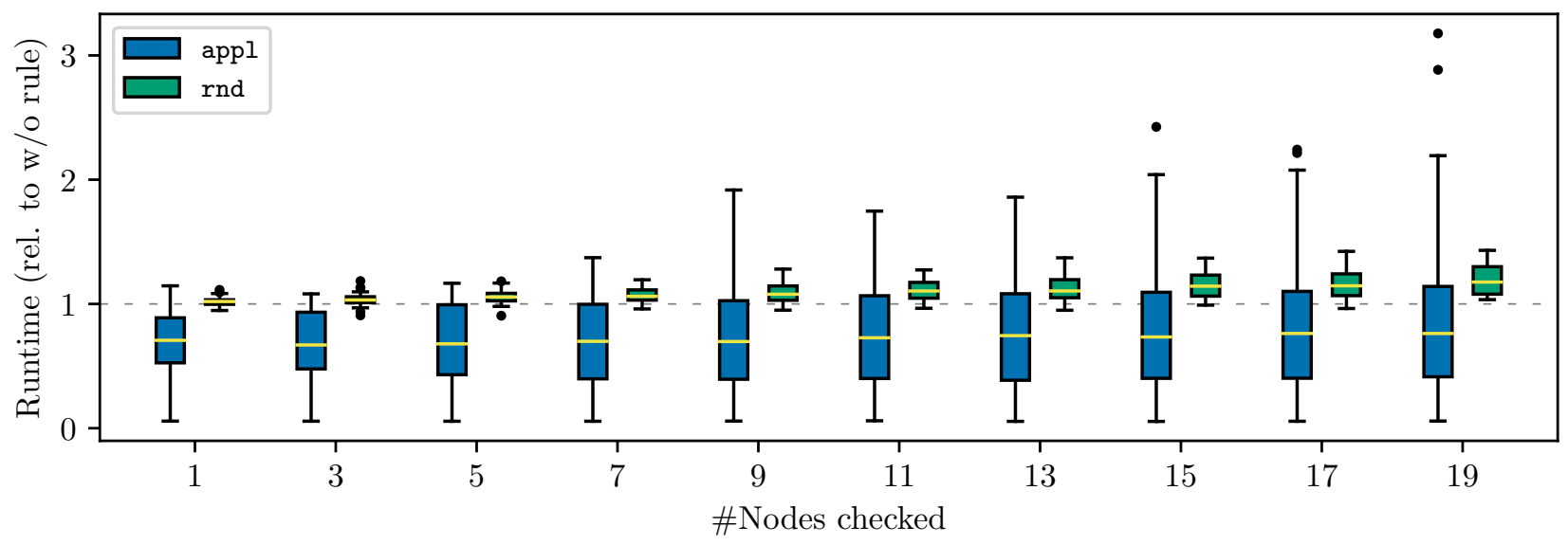

Figure 10: Run time when using different settings for the costly discard packing from scratch rule. All values are relative to the run time when that rule is disabled.

[1] Faisal N. Abu-Khzam. A kernelization algorithm for dhitting set. Journal of Computer and System Sciences, 76(7):524-531, 2010. doi:10.1016/j.jcss.2009.09. 002

[2] Diogo V Andrade, Mauricio GC Resende, and Renato $\mathrm{F}$ Werneck. Fast local search for the maximum independent set problem. Journal of Heuristics, 18(4):525-547, 2012.

[3] Johann Birnick, Thomas Bläsius, Tobias Friedrich, Felix Naumann, Thorsten Papenbrock, and Martin Schirneck. Hitting set enumeration with partial information for unique column combination discovery. Proceedings of the VLDB Endowment, 13(12):2270-2283, 2020. doi:10.14778/3407790.3407824

[4] Thomas Bläsius, Philipp Fischbeck, Tobias Friedrich, and Martin Schirneck. Understanding the effectiveness of data reduction in public transportation networks. In Algorithms and Models for the Web Graph, pages 87-101. Springer International Publishing, 2019. doi: 10.1007/978-3-030-25070-6_7

[5] Sebastian Böcker, Sebastian Briesemeister, Quang Bao Anh Bui, and Anke Trub. A fixed-parameter approach for weighted cluster editing. In Proceedings of the 6th Asia-Pacific Bioinformatics Conference, 2007. doi:10.1142/9781848161092_0023

[6] Michele Borassi, Pierluigi Crescenzi, and Michel Habib. Into the square: On the complexity of some quadratictime solvable problems. Electronic Notes in Theoretical Computer Science, 322:51-67, 2016. doi:10.1016/j. entcs.2016.03.005

[7] Ljiljana Brankovic and Henning Fernau. Parameterized approximation algorithms for hitting set. In $A p$ proximation and Online Algorithms (WAOA 2011), pages 63-76, 2012.

[8] Alberto Caprara, Paolo Toth, and Matteo Fischetti. Algorithms for the set covering problem. Annals of
Operations Research, 98(1/4):353-371, 2000. doi:10. 1023/a: 1019225027893

[9] Danilo Carastan-Santos, Raphael Y. de Camargo, David C. Martins, Siang W. Song, and Luiz C.S. Rozante. Finding exact hitting set solutions for systems biology applications using heterogeneous GPU clusters. Future Generation Computer Systems, 67:418-429, 2017. doi:10.1016/j.future.2016.02. 009.

[10] Danilo Carastan-Santos, Raphael Yokoingawa De Camargo, David Correa Martins, Siang Wun Song, Luiz Carlos Silva Rozante, and Fabrizio Ferreira Borelli. A multi-GPU hitting set algorithm for GRNs inference. In 2015 15th IEEE/ACM International Symposium on Cluster, Cloud and Grid Computing, pages 313-322, 2015. doi:10.1109/CCGrid.2015.29

[11] Karthekeyan Chandrasekaran, Richard Karp, Erick Moreno-Centeno, and Santosh Vempala. Algorithms for implicit hitting set problems. In Proceedings of the Annual ACM-SIAM Symposium on Discrete Algorithms (SODA 2011), pages 614-629, 2011. doi: 10.1137/1.9781611973082.48

[12] Kenneth L. Clarkson and Kasturi Varadarajan. Improved approximation algorithms for geometric set cover. Discrete \& Computational Geometry, 37:43-58, 2007.

[13] Thomas H Cormen, Charles E Leiserson, Ronald L Rivest, and Clifford Stein. Introduction to algorithms. MIT press, 2009.

[14] Graham Cormode, Howard Karloff, and Anthony Wirth. Set cover algorithms for very large datasets. In Proceedings of the 19th ACM International Conference on Information and Knowledge Management (CIKM 2010), pages 479-488, 2010. doi:10.1145/1871437. 1871501

[15] Johan de Kleer. Hitting set algorithms for model- 
based diagnosis. In 22nd International Workshop on Principles of Diagnosis, 2011.

[16] Irit Dinur and David Steurer. Analytical approach to parallel repetition. In Forty-Sixth Annual ACM Symposium on Theory of Computing (STOC 2014), pages 624-633, 2014. doi:10.1145/2591796.2591884.

[17] Henning Fernau. Parameterized algorithms for hitting set: The weighted case. In Algorithms and Complexity (CIAC 2006), pages 332-343, 2006.

[18] Andrew Gainer-Dewar and Paola Vera-Licona. The minimal hitting set generation problem: Algorithms and computation. SIAM Journal on Discrete Mathematics, 31(1):63-100, 2017. doi:10.1137/15m1055024

[19] Lars Gottesbüren, Michael Hamann, Philipp Schoch, Ben Strasser, Dorothea Wagner, and Sven Zühlsdorf. Engineering Exact Quasi-Threshold Editing. In 18th International Symposium on Experimental Algorithms (SEA 2020), volume 160 of Leibniz International Proceedings in Informatics (LIPIcs), pages 10:1-10:14, 2020. doi:10.4230/LIPICs.SEA.2020.10

[20] Tal Grossman and Avishai Wool. Computational experience with approximation algorithms for the set covering problem. European Journal of Operational Research, 101(1):81-92, 1997. doi:10.1016/ s0377-2217(96)00161-0

[21] Gurobi Optimization, LLC. Gurobi Optimizer Reference Manual, 2021. URL: https://www.gurobi.com

[22] Sepp Hartung and Holger H. Hoos. Programming by optimisation meets parameterised algorithmics: A case study for cluster editing. In Learning and Intelligent Optimization, pages 43-58. Springer International Publishing, 2015.

[23] Trey E. Ideker, Vesteinn Thorsson, and Richard M. Karp. Discovery of regulatory interactions through perturbation: Inference and experimental design. In Pacific Symposium on Biocomputing, pages 302-313, 2000.

[24] Richard M. Karp. Reducibility among combinatorial problems. In Complexity of Computer Computations, pages 85-103. Springer US, 1972. doi:10.1007/ 978-1-4684-2001-2_9

[25] Erick Moreno-Centeno and Richard M. Karp. The implicit hitting set approach to solve combinatorial optimization problems with an application to multigenome alignment. Operations Research, 61(2):453-468, 2013. doi:10.1287/opre.1120.1139

[26] Keisuke Murakami and Takeaki Uno. Efficient algorithms for dualizing large-scale hypergraphs. Discrete Applied Mathematics, 170:83-94, 2014. doi:10.1016/ j.dam.2014.01.012

[27] Sven Rahmann, Tobias Wittkop, Jan Baumbach, Marcel Martin, Anke Truß, and Sebastian Böcker. Exact and heuristic algorithms for weighted cluster editing. In Computational Systems Bioinformatics, 2007. doi:10.1142/9781860948732_0040

[28] Raymond Reiter. A theory of diagnosis from first principles. Artificial Intelligence, 32(1):57-95, 1987. doi:10.1016/0004-3702(87)90062-2
[29] Iztok Savnik. Index data structure for fast subset and superset queries. In Availability, Reliability, and Security in Information Systems and HCI, pages 134148. Springer Berlin Heidelberg, 2013. doi:10.1007/ 978-3-642-40511-2_10.

[30] Lei Shi and Xuan Cai. An exact fast algorithm for minimum hitting set. In 2010 Third International Joint Conference on Computational Science and Optimization. IEEE, 2010. doi:10.1109/cso.2010.240.

[31] Steven S. Skiena. The Algorithm Design Manual. Springer International Publishing, 2020. doi:10. 1007/978-3-030-54256-6

[32] Petr Slavík. A tight analysis of the greedy algorithm for set cover. Journal of Algorithms, 25(2):237-254, 1997. doi:10.1006/jagm.1997.0887.

[33] René van Bevern and Pavel V. Smirnov. Optimalsize problem kernels for d-hitting set in linear time and space. Information Processing Letters, 163:105998, 2020. doi:10.1016/j.ipl.2020.105998.

[34] Karsten Weihe. Covering trains by stations or the power of data reduction. Algorithms and Experiments, ALEX, pages 1-8, 1998.

[35] Ćendić Lazović Bojana. A genetic algorithm for the minimum hitting set. Scientific Publications of the State University of Novi Pazar Series A: Applied Mathematics, Informatics and mechanics, 6(2):107117, 2014. 


\section{A Missing Proofs from Section 2}

Lemma 2.1. The sum-over-packing dominates the sumdegree and the packing bound. The sum-degree bound dominates the max-degree bound and is dominated by the efficiency bound.

Proof. We first show that the sum-over-packing bound dominates the sum-degree, and the packing bound. The packing bound cannot be larger because the sum-overpacking bound adds a non-negative number to it. Also the sum-over-packing bound over an empty packing is exactly the sum-degree bound.

The max-degree bound can be expressed as the smallest $k$ for which $\sum_{i=1}^{k} d_{1} \geq|\mathcal{F}|$ showing that the sum-degree bound dominates the max-degree bound.

It remains to show that the efficiency bound dominates the sum-degree bound. The sum-degree bound is given by the $k$ highest degree vertices whose degrees sum up to just above $|\mathcal{F}|$. Let $v_{1}, \ldots, v_{k}$ be these vertices sorted descending by degree. We partition the edges of $\mathcal{F}$ into $k$ sets $E_{1}, \ldots, E_{k}$ with the following two properties. First, $\left|E_{i}\right|=\operatorname{deg}\left(v_{i}\right)$ for $i<k$ and $1 \leq\left|E_{k}\right| \leq \operatorname{deg}\left(v_{k}\right)$. Second, the maximum vertex degree for every edge in $E_{i}$ is at most $\operatorname{deg}\left(v_{i}\right)$. Such a partition can be achieved as follows. Assign to $E_{1}$ all edges containing $v_{1}$. For larger $i$, assign to $E_{i}$ all edges that contain $v_{i}$ that have not yet been assigned. Moreover, add further edges arbitrarily, until $E_{i}$ contains $\operatorname{deg}\left(v_{i}\right)$ edges. The first property, concerning the sizes of $E_{i}$, clearly holds. For the second property, observe that in step $i$, all edges containing vertices $v_{1}, \ldots, v_{i-1}$ have already been assigned. Thus, all unassigned edges, and thereby all edges ending up in $E_{i}$, only contain vertices of degree at most $\operatorname{deg}\left(v_{i}\right)$.

With this partition, we get that the efficiency bound is larger than $k-1$, because

$$
\begin{aligned}
\sum_{F \in \mathcal{F}} \min _{v \in F} \frac{1}{\operatorname{deg}(v)} & =\sum_{i=1}^{k} \sum_{F \in E_{i}} \min _{v \in F} \frac{1}{\operatorname{deg}(v)} \\
& \geq \sum_{i=1}^{k} \sum_{F \in E_{i}} \frac{1}{\operatorname{deg}\left(v_{i}\right)} \\
& =k-1+\frac{\left|E_{k}\right|}{\operatorname{deg}\left(v_{k}\right)}>k-1,
\end{aligned}
$$

and thus the rounded-up efficiency bound is at least as high as the sum-degree bound.

LEMMA 2.2. The packing bound is incomparable with the max-degree, sum-degree, and efficiency bound. The efficiency bound is incomparable with the sum-overpacking bound.

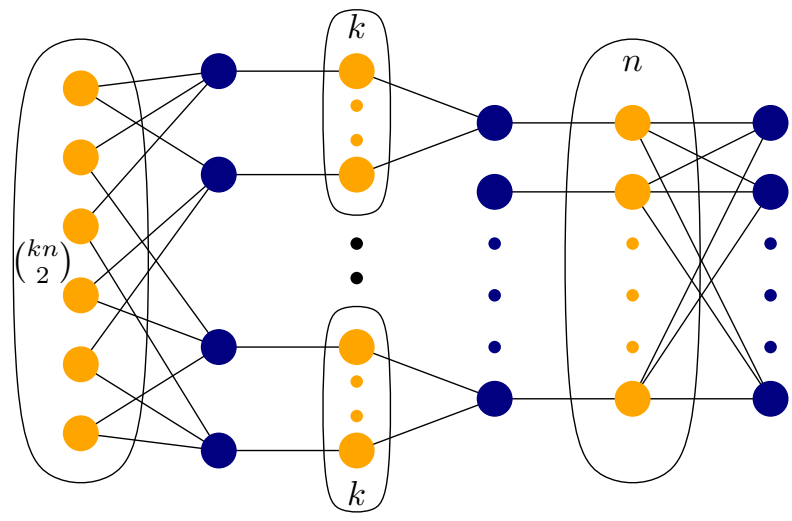

Figure 11: Instance family represented as a bipartite graph. The sum-over-packing bound is worse than efficiency for $k=2$ and efficiency $<$ packing for $k=1$. Edges are blue and vertices are orange.

Proof. We give examples of instances where (1) packing < max-degree, (2) efficiency < packing, and (3) sum-over-packing < efficiency; see Figure 1. With the domination relations from Lemma 2.1 the incomparability of the packing bound follows from the instances where packing $<(1)$ max-degree $\leq$ sum-degree $\leq$ efficiency $<(2)$ packing. Similarly, the second part of the lemma follows from the instances where efficiency $<(2)$ packing $\leq$ sum-over-packing $<(3)$ efficiency.

In the example for (1), there are three vertices $a, b, c$ and three edges $\{a, b\},\{a, c\},\{b, c\}$. Every packing contains at most one edge. In contrast the max-degree bound is $\lceil 3 / 2\rceil=2$.

We give a parametrized example for (2) and (3). The construction is shown in Figure 11. There are three types of edges. In the center, there are $n$ disjoined edges, the center edges, that constitute a maximum packing. To the right there are $n$ edges, the right edges, that share $n$ vertices. To the left are $k \cdot n$ edges, the left edges, that pairwise share a vertex of degree two. Each of the center edges contains one of $n$ high degree vertices shared by the right edges. Each center edge also contains $k$ vertices of degree two that are also contained in one of the $k \cdot n$ left edges.

For $k=1$, the efficiency bound is (for edges from left to right) $n / 2+n /(n+1)+n /(n+1)<n$ while the largest packing, e.g., the center edges, has size $n$. Thus, the efficiency bound can be smaller than the largest packing showing (2).

For $k=2$ the efficiency bound is $2 n / 2+n /(n+$ $1)+n /(n+1)>n$. To prove $(3)$, it remains to show that the sum-over-packing bound is at most $n$ for each possible packing. Note that the argument must hold for each packing and not just maximum packings because 
a maximum packing does not necessarily lead to the highest sum-over-packing bound. If a packing includes a right edge, this prevents the inclusion of all other right edges and all center edges. If a packing includes a left edge, this prevents the inclusion of all other left edges and exactly one center edge. The only disjoined packings possible are either only center edges, one left edge and the rest center edges, or one left and one right edge. Thus, each packing $P$ has size at most $n$ and contains at least $|P|-2$ center edges, which have vertices of degree $n+1$.

Now we show that the sum-over-packing bound is either constant or limited by the packing size. For sufficiently large $n$ and any packing $S$ that is larger than six, the packing contains at least four center edges. The sum-over-packing bound adds nothing to the size of the packing because the maximum degrees of the vertices in four center edges add up to more than $|\mathcal{F}|$. That is, the sum-over-packing bound is $|S|$, because $\sum_{F \in S} \max _{v \in F} \operatorname{deg}(v)>4 n=|\mathcal{F}|$. For any packing $S$ smaller than six, the remaining instance after deleting $S$ and its max-degree vertices still contains $\Omega(n)$ nodes of degree at least $n$. Four of them suffice to obtain a degree sum larger than $|\mathcal{F}|$. Therefore the sum-overpacking bound is at most $|S|+4 \leq 10$. In each case, the sum-over-packing bound is at most $n$, thus smaller than the efficiency bound.

\section{B Additional Evaluation Results}

This section contains additional evaluation results that have been cut from the main paper due to space constraints.

B.1 Search Space and Bounds. Figure 12 shows the size of the search space depending on the instance size and the difference between the initial upper and lower bound. The lack of samples with low search space and instance size is an artifact due to the exclusion of instances that are solved in less than a second.

There appears to be no clear correlation to indicating that instance size reflect difficulty. On the other hand, the bound gap is usually a good estimate for the difficulty of an instance to a given branch-and-bound solver, because the solver lowers this gap during execution and is finished if the difference reaches zero. The random instances exhibit a distinct exponential growth in search space with growing gap.

Figure 13 further explores the difference between lower and upper bounds. The initial upper bounds are already close to the optimum with all but three being less than 1.3 times the optimum. Lower bounds are spread out more. Some instances have lower bounds that are not even a third of the optimum. Interestingly, the instance with with worst upper bound has a perfect lower bound.

Figure 14 shows the progression and gradual lowering of the upper bound during execution. Each individual instance starts in the lower left corner at $(0,0)$ and progresses to the upper right corner at $(100,100)$. Under the assumption that this progression is linear we would get white above and black below the main diagonal. However, the plot can be better described as growing darker from left to right. This verticality means that during a run, upper bounds progress is often achieved all at once. Also the upper bound is surprisingly good with more than $40 \%$ of the instances already having a perfect upper bound to start with. After $25 \%$ of the run time the optimum has been found (100\% progress) for more than $60 \%$ of instances. The remaining $75 \%$ of the run time are used for proving its optimality. 

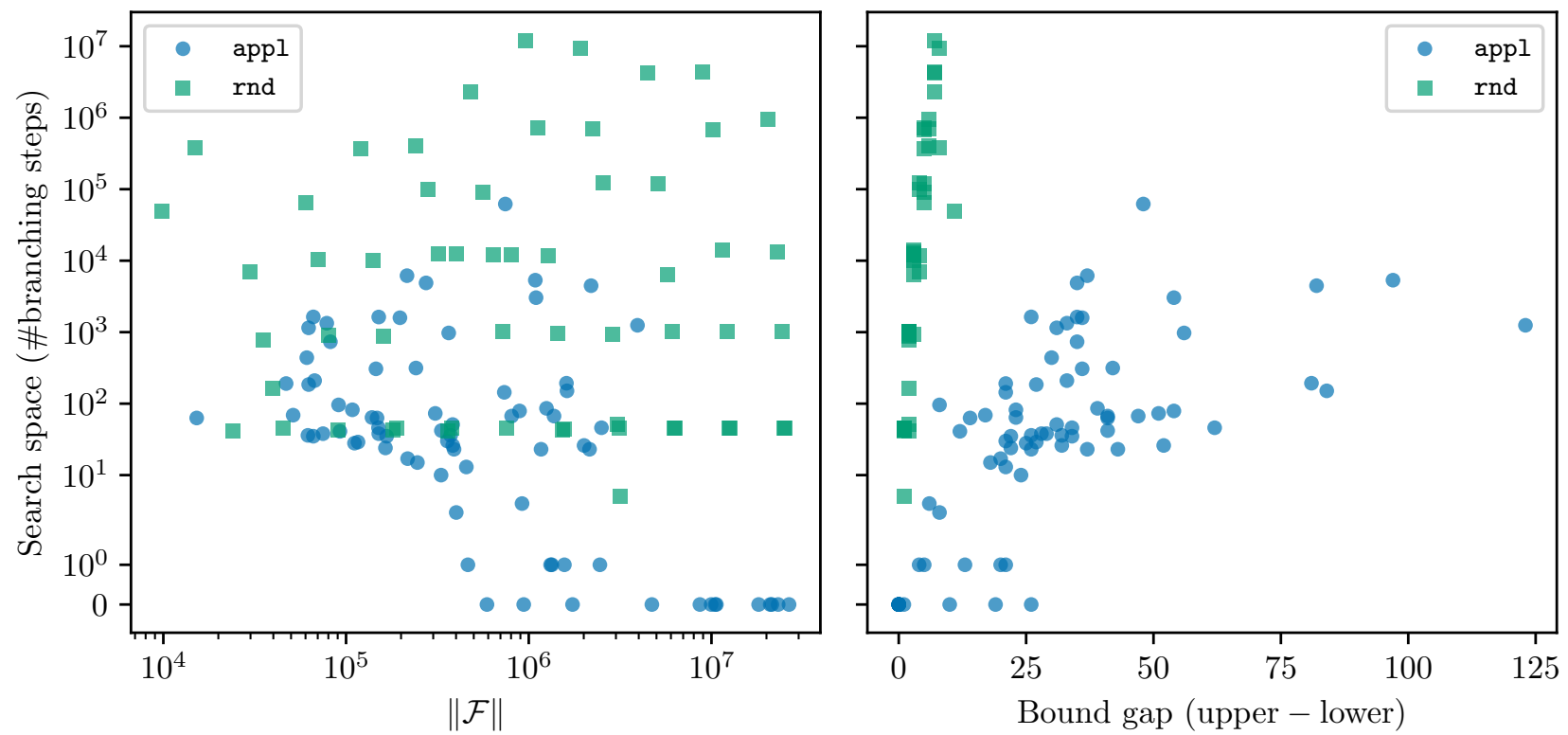

Figure 12: Search space compared to instance size (left) and difference between upper and lower bound (right). Each instance represents one sample. The y-axis is logarithmic with special handling for zero.

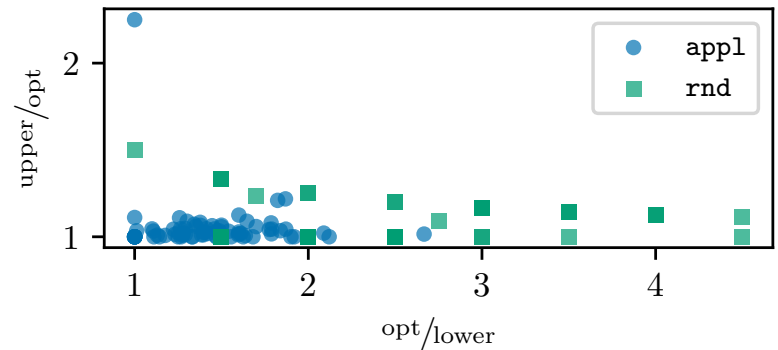

Figure 13: The plot shows the initial lower bound relative to opt on the $\mathrm{x}$-axis and the upper bound relative to opt on the y-axis. Each instance represents one sample.

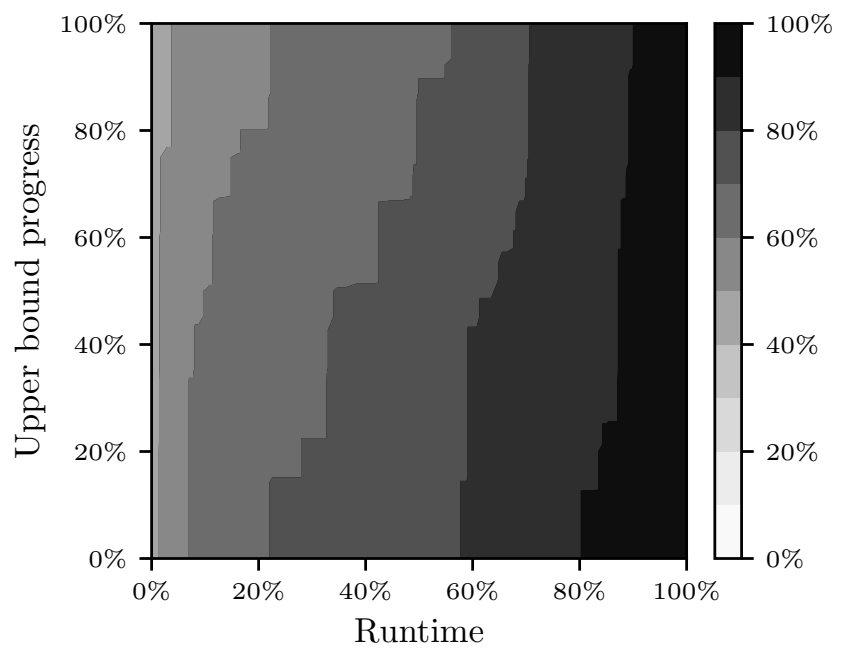

Figure 14: For a given run time and a given amount of progress from the initial upper bound towards opt, how many instances have reached that progress at that time. 